\title{
A Cyclist-Domain-Based Method for Evaluating the Level of Service of a Bicycle Lane
}

\author{
Sheng Zhang $\mathbb{D}$, Jun Liang, and Zhongxiang Huang \\ School of Traffic and Transportation Engineering, Changsha University of Science \& Technology, Changsha 410114, China \\ Correspondence should be addressed to Zhongxiang Huang; mehzx@126.com
}

Received 24 August 2019; Accepted 18 May 2020; Published 8 June 2020

Academic Editor: Alexander Paz

Copyright (c) 2020 Sheng Zhang et al. This is an open access article distributed under the Creative Commons Attribution License, which permits unrestricted use, distribution, and reproduction in any medium, provided the original work is properly cited.

Evaluating the level of service (LOS) of bicycle lanes is essential in planning, designing, and maintaining bicycle infrastructure. This study presents a new concept-the cyclist domain-for characterizing interactions between cyclists and proposes a cyclistdomain-based method for evaluating the LOS of bicycle lanes. Unlike conventional bicycle lane LOS evaluation method, which counts the weighted events numbers and may generate inaccurate results and even misleading decisions, we specified four types of overlapping cyclist domains to characterize potential impacts in various riding events. The cyclist domain influence ratio (CDIR) derived from cycling trajectories is used to measure the negative impact between cyclists. We then propose an LOS evaluation method for bicycle lanes based on CDIRs. The user comfort is defined as the sum of products of CDIRs and respective durations. The bicycle lane LOS is then evaluated by the average comfort of all users. The case study shows that the proposed method can efficiently and effectively evaluate the bicycle lane LOS.

\section{Introduction}

Bicycling is a clean, sustainable mode of transport that helps riders efficiently fulfill multimodal trips. Sims et al. [1] and Ermagun and Levinson [2] refer to cycling as active travel, in which users are provided with an opportunity to accomplish a portion of their recommended daily physical activity. The past decade has witnessed the rapid worldwide expansion of bicycle transport, driven by bike-sharing companies such as Mobike and Ofo. In recent years, bicycling transport has attracted increasing attention from researchers and practitioners. In theoretical side, quite a few academic developments are made, e.g., bike-repositioning problem (Angeloudis et al. [3]; Forma et al. [4]; Li et al. [5]; and Ho and Szeto [6]), geometric design of bike paths (Jia et al. [7]), bicycling network design (Duthie and Unnikrishnan [8]), and layout of bike-sharing rental stations (Duan et al. [9] and Chen and Sun [10]). In practical side, nowadays, many metropolitan areas have launched a new round of construction projects of bicycle facility to accommodate increasing cycling demands (Faghih-Imani and Eluru [11]).
The level of service (LOS) of bicycle lanes is vital for quantitatively evaluating the performance of bicycle lanes. It is also used for guiding the infrastructure planning and the operation of bicycle transport service. For example, transport sectors and designers need to identify relationships between the LOS and bicycle lane's geometrics (e.g., lane width) before putting forward specific planning schemes for cycling transport. When managing a safe and effective cycling transport system, the authority should also know the impact of external traffic conditions on the LOS of bicycle lanes.

A vast body of literature focused on identifying relationships between operational effects of bicycle lanes and influencing factors such as lane width and traffic condition by using statistical analysis. For example, Landis [12], based on survey responses from bicyclists, introduced a statistically calibrated LOS model to reveal that pavement conditions and lane markings were crucial influential factors for the LOS of a bicycle lane. Kang and Lee [13] developed an evaluation model and a set of LOS criteria for South Korea, which incorporated user satisfaction and other factors (including lane width, road type, the number of encounters, 
and the number of lanes near intersections). Liang et al. [14] proposed an entropy-based model to determine the LOS of bicycle lanes in China and gave a LOS classification table.

Cyclists' perception on comfort, convenience, and security is viewed as one critical indicator for the LOS of bicycle lanes. Some researchers started from this perspective to estimate the LOS by using hindrances encountered during riding. Botma [15] defined the hindrance as the fraction of users over a $1.0-\mathrm{km}$ path impeded from passing and meeting manoeuvres. He thus proposed a criterion for bicycle-lane LOS as the per cent of the number of hindrances in the number of users passed (moving in the same direction) plus the number of users encountered (moving in the opposite direction) by the bicyclist. This criterion is time related of the duration which a bicyclist involved in an event. This leads to computational intractability and also restricts its application in real world.

Hummer et al. [16] made an extension to estimate the LOS of bicycle lanes in the US by using the number of passing manoeuvres and contributed to the formation of wide-applied method collected in Highway Capacity Manual (HCM), 2010 [17]. They presented a bicycle lane LOS score model incorporating various variables of cyclists' perceptions. These variables include the number of times that a cyclist meets with others per minute, the number of times per minute on average that a bicyclist passes or is delayed in passing others, and the presence of a centre line and path width. A regression model of user responses to video clips that record the use of a variety of off-street bicycle facilities is developed to calculate the LOS score of the bicycle lanes. The HCM method takes into account the amount of active passing and meetings, the probability of delayed passing and hindrances.

The HCM method may generate inaccurate results and even misleading decisions for the following two reasons. Firstly, not every riding event brings hindrance to cyclists. In this sense, the HCM method may overestimate the impact of hindrance. Secondly, a particular event exerts on different negative impacts on distinct cyclists. Under this case, a regression model with a set of fixed weighted parameters cannot well characterize cyclists' perception from a microscopic view. Therefore, we need to seek a satisfactory solution to evaluate the LOS of bicycle lanes.

The main challenge of accurately evaluating the LOS of bicycle lanes stems from the high freedom of microscopic cycling behaviours. Cycling traffic exhibits obvious disparities from conventional vehicle traffic in that cycling trajectories are not strictly subject to bicycle lanes. Therefore, the fundamental diagram in terms of capacity-volume-speed relationship of cycling traffic may not hold and the LOS evaluation method for highways may not be applicable for bicycle lanes. Although a few existing studies stressed the importance of considering cyclists' perceptions in estimating the LOS of bicycle lanes, the methods are not able to characterize the perceptions well due to the absence of taking into microscopic riding behaviours.

The concept of domain offers a chance to appropriately describe cyclists' perceptions and enables us to develop a more realistic and effective bicycle lane LOS evaluation method because the changes of cyclist's domain directly and accurately reflect user's real-time psychology and perception. Sommer [18] puts forward a concept of personal space, which provides a solution to characterize people's perception. The personal space is similar to a variable bubble around cyclist upon external traffic environment. Any invasion of or interference with one's personal space would bring about anxiety. Kim et al. [19] proposed a behaviouraltheory-based approach to better assess pedestrian LOSs for sidewalks using the concept of personal space and pedestrian evasive movements. With the use of a separate validation sample, the revised LOS obtained was found to be more consistent with pedestrians' perceptions than the levels prescribed by the Korean HCM. The concept of domain is initiatively used in ship navigation for ship collision avoidance. Goodwin [20] is the first to give the definition for ship domain, which is stated as "the surrounding effective waters which the navigator of a ship wants to keep clear of other ships or fixed objects." Any violation of ship domain is interpreted as a threat to navigational safety.

As we know, there are three types of cycling events, namely, passing, meeting, and following. An event may drive a cyclist to change her/his actions, especially cyclists feel anxious when others are approaching. Furthermore, cyclists' velocities would be changed in different extents according to the event type. The degree of a cyclist's negative perception can be described by the change of her/his psychological space, and the private space has been invaded. In spite of ship domain, we define cyclist's psychological space as the cyclist domain.

In this paper, we propose a cyclist-domain-based method for evaluating the LOS of bicycle lanes. Our study makes substantial contributions to the literature of bicycle transport by building a cyclist-domain-based method for evaluating the LOS of bicycle lanes and proposing LOS classification criteria for bicycle lane planning. The proposed evaluation method could well take into account the changes of cyclists' psychology and perception while cycling and generate more accurate evaluation results. The reminder of this paper is organized as follows. We shall devote Section 2 to introducing a framework of our proposed LOS evaluation method. We then present in Section 3 the cyclist-domain-based method. Case study and data analysis are provided in Section 4. Section 5 concludes the paper.

\section{Conceptual Framework}

In spite of the theory of personal space, we define the user's personal space in a bicycle lane as the cyclist domain, which could well describe cyclists' perception. Invasion of a cyclist's domain will lead to negative effect to her. The invaded region of a cyclist domain is the overlapping part of two neighbouring cyclists.

Figure 1 provides an example of the mutual interference process between two cyclists. Two cycling trajectories are shown in the figure (the red dashed and black solid lines represent Users 1 and 2, respectively). The ellipses are used to represent the real-time sizes of users' cyclist domains. The 


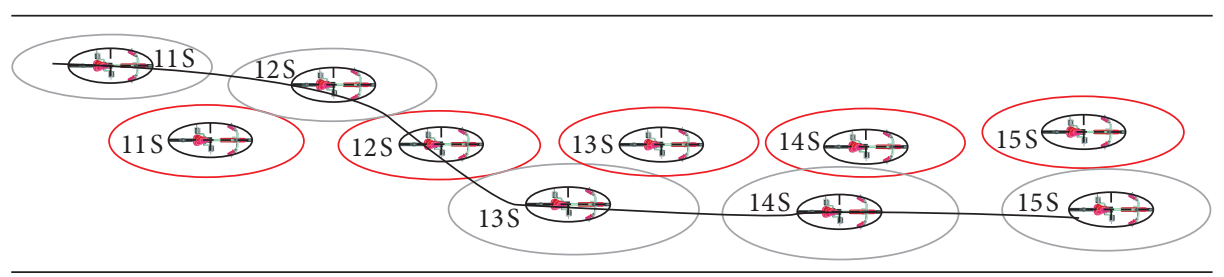

Figure 1: A domain-based description of a cycling event.

number on each ellipse denote time label. For example, the number 12 indicates that cyclist arrives at the focal position at 12 th time step. Both cyclists pass the segment of bicycle lane within 5 seconds. The real-time size of the ellipse (cyclist domain) would be to a great extent determined by the cyclist's instantaneous velocity.

As shown in Figure 1, the interference between the two cyclists is recorded as a panel data. Two individuals' cyclist domains are bounded at the 12th time step, maintained overlapped in the 12th-15th time steps, and separated after then. Evidently, mutual interference (also called domain invasion) occurs several times.

Figure 2 displays the workflows of our proposed LOS evaluation method for bicycle lanes. The overlapping and the change in the area of cyclists' domains reflect changes in user perception. After tracking cycling trajectories, the area of overlapping cyclists' domains can be calculated using the definite integral method. We here define CDIR to measure the changing degree of a cyclist's perception in a single cycling event. We define comfort level to measure the changes of a cyclist's perception in the entire cycling process on the target bicycle lane. The average comfort level of all cyclists could serve as the bicycle lane LOS score. A method based on cyclist domain clearly shows how each cyclist's domain evolves and cyclists interfere with each other.

The procedures are elaborated as below:

(i) Step 1 (data preparation): the cycling trajectories can be extracted using image identification software, and the time and labour cost could be saved. Specifically, the evaluation period should be consistent with the traffic signal cycle of the upstream intersection. According to the actual situation, we suggest that a bicycle lane should be counted for several different traffic signal cycle during the peak hour to establish the LOS.

(ii) Step 2 (draw point cloud graphs): points in the cloud graphs describe the relative positions of adjacent cyclists. By regression of these relative position, we can get the cyclist domain model.

(iii) Step 3: calculate the speed-dependent cyclist domains.

(iv) Step 4: compute the overlapping area of cyclist domains for each user.

(v) Step 5: compute the CDIR and the comfort level of each user.

(vi) Step 6: evaluate the LOS of target bicycle lane.

\section{Methodology}

\subsection{The Method to Estimate Cyclist Domain}

3.1.1. Definition of Cyclist Domain. In this paper, cyclist domain is defined as the minimum safe distance that a cyclist maintains sufficient reaction time to events and keeps riding comfortably, in the cyclist's perception. Similar to Horowitz et al. [21], given body-buffer zones and cycling behaviour characteristics, we also assume that the profile of a cyclist domain is an ellipse with its semimajor axis parallel to the bicycle lane. Figure 3 provides an example for the velocitydependent cyclist domain. When cyclists travel with speed $v$, the corresponding radius of the semimajor axis of the cyclist domain ellipse is longitudinal separation denoted by $a$; the radius of the semiminor axis of the ellipse is horizontal separation denoted by $b$.

3.1.2. Regression Model to Obtain Cyclist Domains. In this section, we introduce how to calculate velocity-dependent cyclist domains by using the regression method. The data were obtained from a released cycling traffic video filmed at the intersection of Vredenburg, Lange Viestraat, and Sint Jacobsstraat, on the edge of Utrecht Central Station. The video was taken during morning peak hour in April 2014. Data of 20 cyclists were extracted from the video in 0.5 second intervals, including real-time speed and adjacent cyclists' polar coordinates.

The procedures to calculate cyclist domain are briefly presented here. We first draw point cloud graphs based on the extracted cycling trajectories, then obtain domain characteristic variables from the point cloud graphs, and make a regression analysis to filter the best one according to the goodness of fitting.

Figure 4 shows the point cloud graphs of different speeds based on 242 data points. The cycling direction is set parallel to the $x$-axis. The cyclist is at the origin. The horizontal and vertical coordinates represent the semimajor axis $a$ and the semiminor axis $b$ of the cycling space, respectively. Both $a$ and $b$ are in meters.

Based on the point cloud data, the elliptic curves for different speeds can be fitted by using ellipse fitting. The observed data are collected in Table 1.

The curve estimation method in SPSS 22.0 was used to derive the formulas of $a$ and $b$ with the independent variable of speed, based on the data given in Table 1. Curve estimation results are shown in Tables 2 and 3 and also plotted in Figure 5 for an intuitive illustration. As shown in the tables and figures, of the ten models tested, the quadratic 


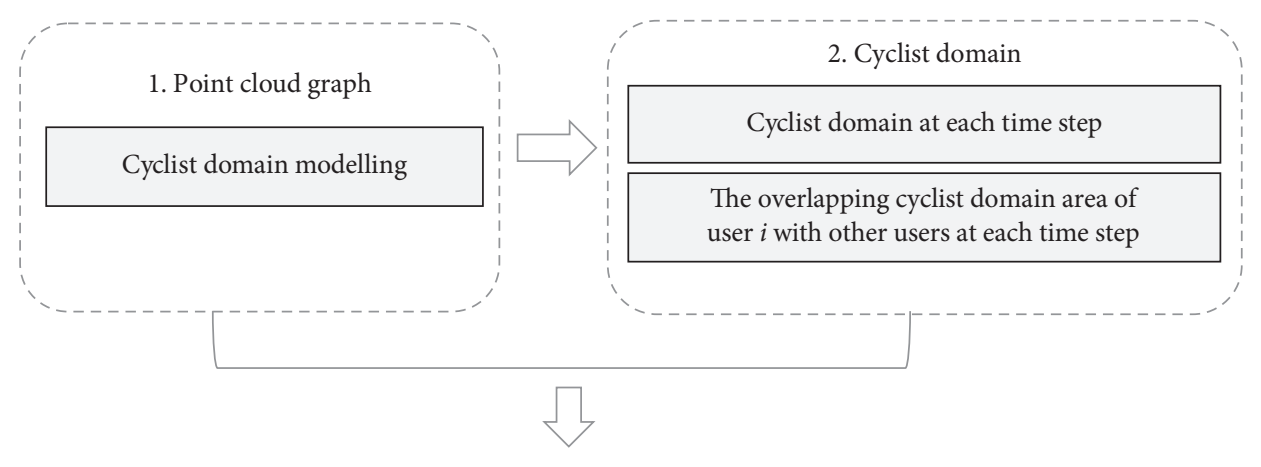

3. CDIR of user $i$

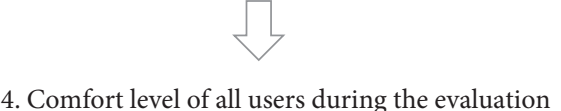

4. Comfort level of all users during the evaluation

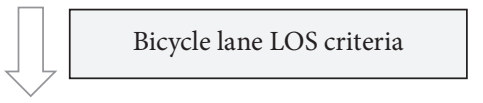

5. Bicycle lane LOS

FIgURE 2: Study framework.

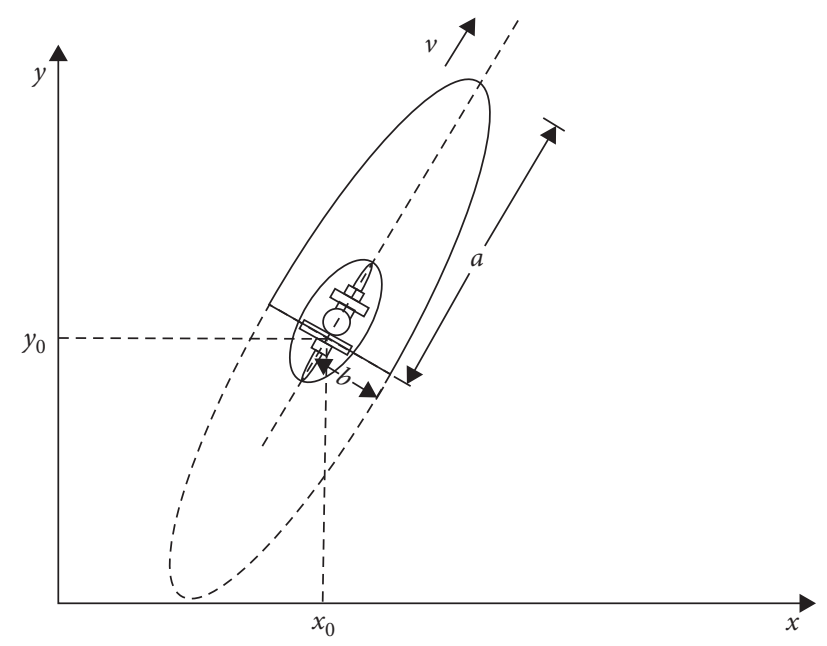

Figure 3: Schematic diagram of the cyclist domain.

Equation showed the best fitting goodness and higher accuracy for semimajor axis $a$ and semiminor axis $b$.

Based on the goodness-of-fit tests, the quadratic Equation was used to fit parameters $a$ and $b$ in the cyclist domain model:

$$
\left\{\begin{array}{l}
a=2.905-1.185 v+0.251 v^{2} \\
b=-0.059+0.428 v-0.035 v^{2}
\end{array}\right.
$$

Meanwhile, the standard elliptic equations for cyclist domain are given by

$$
\frac{\left(x_{i}-x_{i, t}\right)^{2}}{\left(2.905-1.185 v_{i, t}+0.251 v_{i, t}^{2}\right)^{2}}+\frac{\left(y_{i}-y_{i, t}\right)^{2}}{\left(-0.059+0.428 v_{i, t}-0.035 v_{i, t}^{2}\right)^{2}}=1 .
$$

3.2. Calculating the Overlapping Area between Cyclist Domains. Cyclists may experience a variety of events including active passing, meeting, following, and cycling sideby-side. The cyclist domains change over time or have overlapping attributes during different events. Table 4 lists four kinds of cyclist domain overlapping cases in a bicycle lane.

The relative position between neighbouring cyclists and their overlap appearing in all events are summarised in the four situations shown in Figure 6:

The overlapping domain area reflects the cyclist's perception and can be calculated using the definite integral method.

The area of a side-overlapping region of two cyclist domains is calculated by 


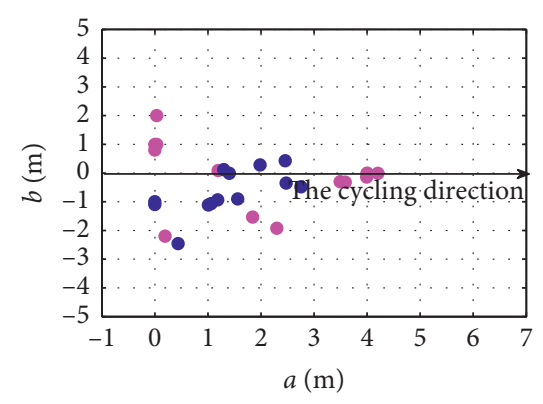

(a)

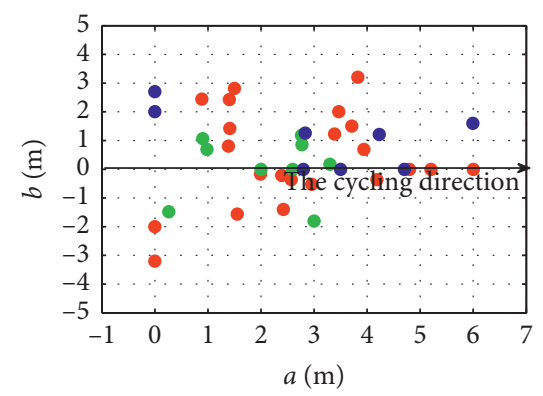

(d)

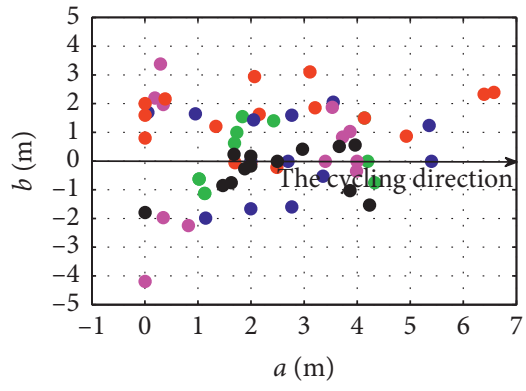

(b)

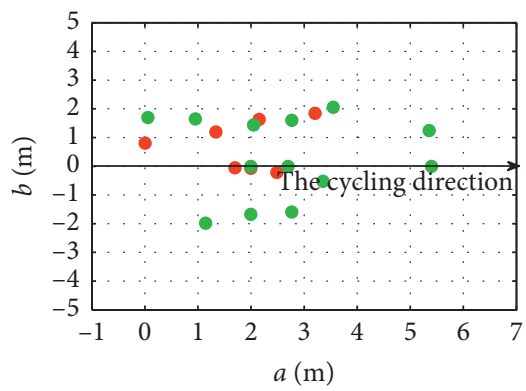

(e)

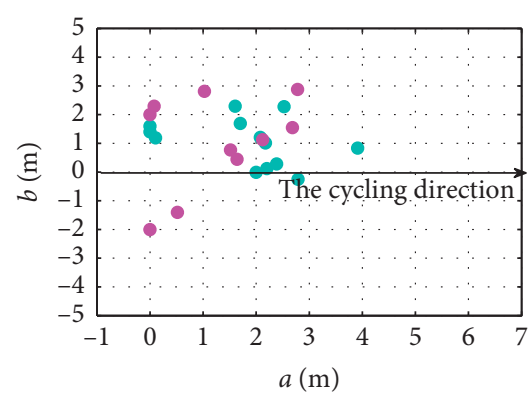

(c)

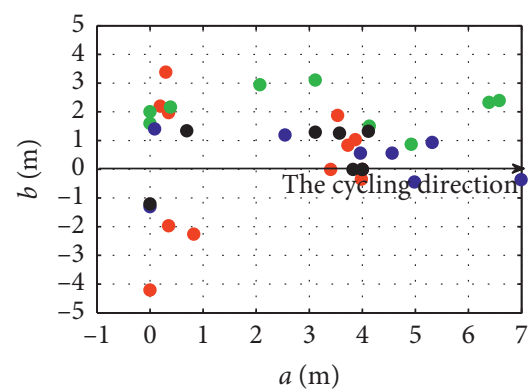

(f)

Figure 4: Point cloud graphs at different speeds with cycling speed (a) $2.5 \mathrm{~m} / \mathrm{s}$, (b) $3 \mathrm{~m} / \mathrm{s}$, (c) $3.5 \mathrm{~m} / \mathrm{s}$, (d) $4 \mathrm{~m} / \mathrm{s}$, (e) $4.5 \mathrm{~m} / \mathrm{s}$, and (f) $5 \mathrm{~m} / \mathrm{s}$.

TABle 1: Observed cyclist domain data.

\begin{tabular}{lccc}
\hline No. & Speed $(\mathrm{m} / \mathrm{s})$ & Longitudinal separation $a(\mathrm{~m})$ & Horizontal separation $b(\mathrm{~m})$ \\
\hline 1 & 2.0 & 1.44 & 0.68 \\
2 & 2.2 & 1.42 & 0.72 \\
3 & 2.4 & 1.58 & 0.75 \\
4 & 2.6 & 1.61 & 0.86 \\
5 & 2.8 & 1.66 & 0.85 \\
6 & 3.0 & 1.70 & 0.88 \\
7 & 3.2 & 1.74 & 0.91 \\
8 & 3.4 & 1.77 & 0.95 \\
9 & 3.6 & 1.82 & 1.06 \\
10 & 3.8 & 1.95 & 1.08 \\
11 & 4.0 & 2.11 & 1.11 \\
12 & 4.2 & 2.39 & 1.16 \\
13 & 4.4 & 2.51 & 1.17 \\
14 & 4.6 & 2.64 & 1.22 \\
15 & 4.8 & 3.07 & 1.18 \\
& 5.0 & 3.37 & 1.16 \\
\hline
\end{tabular}

TABLE 2: Model summary and parameter estimates of $a$ on $v$.

Dependent variable: $a$ independent variable is speed $v$

\begin{tabular}{lccccccc} 
Equation & \multicolumn{9}{c}{ Model summary } & \multicolumn{3}{c}{ Parameter estimates } \\
& $R^{2}$ & $\mathrm{~F}$ & $\mathrm{df1}$ & $\mathrm{df2}$ & Sig. & Constant & $b_{1}$ \\
\hline Linear & 0.867 & 91.611 & 1 & 14 & 0.000 & 0.041 & 0.574 \\
Logarithmic & 0.784 & 50.933 & 1 & 14 & 0.000 & -0.154 & 1.812 \\
Inverse & 0.687 & 30.777 & 1 & 14 & 0.000 & 3.680 & -5.288 \\
Quadratic & 0.979 & 307.279 & 2 & 13 & 0.000 & 2.905 & -1.185 \\
Compound & 0.929 & 182.523 & 1 & 14 & 0.000 & 0.775 & 0.251 \\
Power & 0.863 & 88.188 & 1 & 14 & 0.000 & 0.698 & 0.858 \\
S & 0.778 & 48.923 & 1 & 14 & 0.000 & 1.465 & -2.538 \\
Growth & 0.929 & 182.523 & 1 & 14 & 0.000 & -0.255 & 0.268 \\
Exponential & 0.929 & 182.523 & 1 & 14 & 0.000 & 0.775 & 0.268 \\
Logistic & 0.929 & 182.523 & 1 & 14 & 0.000 & 1.290 & 0.765 \\
\hline
\end{tabular}


TABLe 3: Model summary and parameter estimates of $b$ on $v$.

\begin{tabular}{|c|c|c|c|c|c|c|c|c|}
\hline \multicolumn{9}{|c|}{ Dependent variable: $b$ independent variable is speed $v$} \\
\hline \multirow{2}{*}{ Equation } & \multicolumn{5}{|c|}{ Model summary } & \multicolumn{3}{|c|}{ Parameter estimates } \\
\hline & $R^{2}$ & $\mathrm{~F}$ & df1 & df 2 & Sig. & Constant & $b_{1}$ & $b_{2}$ \\
\hline Linear & 0.945 & 242.782 & 1 & 14 & 0.000 & 0.338 & 0.184 & \\
\hline Logarithmic & 0.963 & 363.252 & 1 & 14 & 0.000 & 0.232 & 0.618 & \\
\hline Inverse & 0.946 & 245.190 & 1 & 14 & 0.000 & 1.573 & -1.910 & \\
\hline Quadratic & 0.968 & 197.616 & 2 & 13 & 0.000 & -0.059 & 0.428 & -0.035 \\
\hline Compound & 0.932 & 192.151 & 1 & 14 & 0.000 & 0.489 & 1.215 & \\
\hline Power & 0.965 & 390.283 & 1 & 14 & 0.000 & 0.434 & 0.659 & \\
\hline S & 0.965 & 391.503 & 1 & 14 & 0.000 & 0.601 & -2.055 & \\
\hline Growth & 0.932 & 192.151 & 1 & 14 & 0.000 & -0.716 & 0.195 & \\
\hline Exponential & 0.932 & 192.151 & 1 & 14 & 0.000 & 0.489 & 0.195 & \\
\hline Logistic & 0.932 & 192.151 & 1 & 14 & 0.000 & 2.045 & 0.823 & \\
\hline
\end{tabular}

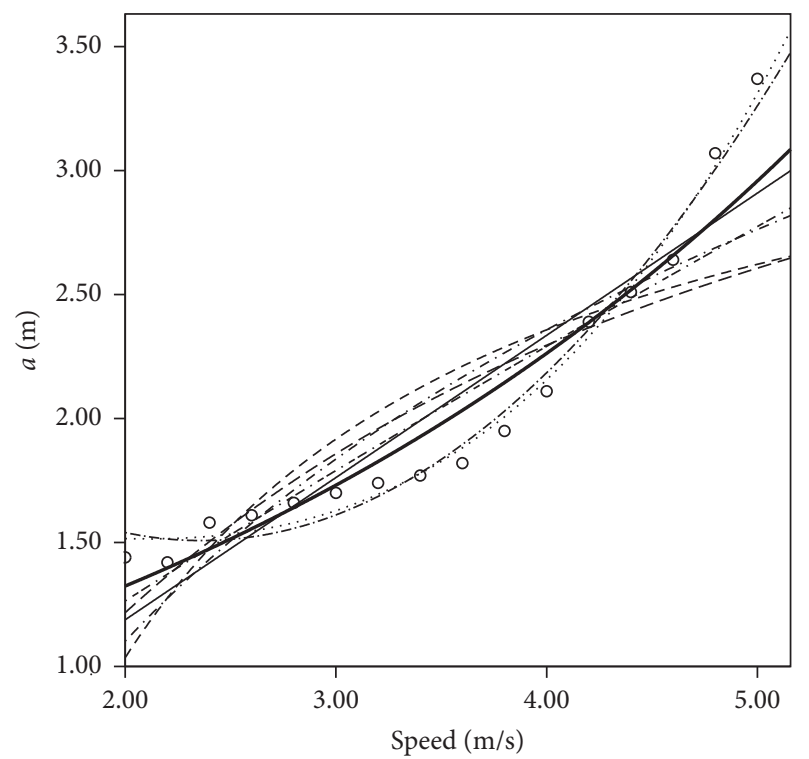
- Observed
- Linear
...- Logarithmic
- - - Inverse
..... Quadratic
Cubic

$$
\begin{aligned}
& \text { — Compound } \\
& \text {.-. Power } \\
& \text {--. S } \\
& \text {-... Growth } \\
& \text {-.- Exponential } \\
& \text {.... Logistic }
\end{aligned}
$$

(a)

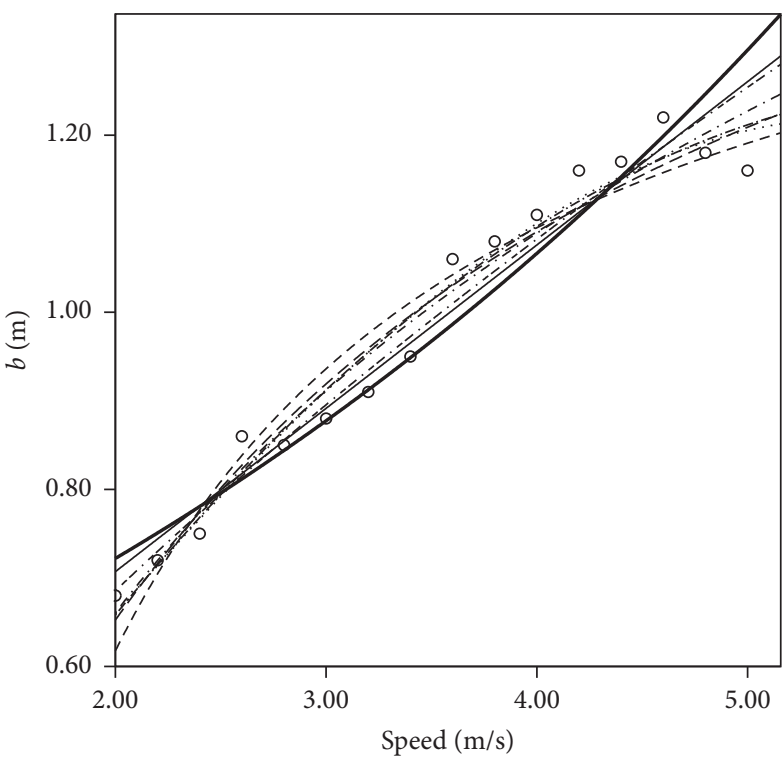
- Observed
— Linear
-. . - Logarithmic
- - - Inverse
-.... Quadratic Cubic

$$
\begin{aligned}
& \text { - Compound } \\
& \text {.-. Power } \\
& \text {--. S } \\
& \text {-... Growth } \\
& \text {-.-. Exponential } \\
& \text {..-. Logistic }
\end{aligned}
$$

(b)

FIgURE 5: Curve estimation.

$$
S_{t, i, j}=\left|\int_{x_{i, j, t, 1}}^{x_{i, j, 2}}\left(y_{i, t} \pm \frac{b_{i, t}}{a_{i, t}} \sqrt{a_{i, t}^{2}-\left(x-x_{i, t}\right)^{2}}\right)-\left(y_{j, t} \pm \frac{b_{j, t}}{a_{j, t}} \sqrt{a_{j, t}^{2}-\left(x-x_{j, t}\right)^{2}}\right) \mathrm{d} x\right| .
$$

The area of a longitudinal-overlapping region of two cyclist domains is expressed by

$$
S_{t, i, j}=\left|\int_{y_{i, j, t, 1}}^{y_{i, j, t, 2}}\left(x_{i, t} \pm \frac{a_{i, t}}{b_{i, t}} \sqrt{b_{i, t}^{2}-\left(y-y_{i, t}\right)^{2}}\right)-\left(x_{j, t} \pm \frac{a_{j, t}}{b_{j, t}} \sqrt{b_{j, t}^{2}-\left(y-y_{j, t}\right)^{2}}\right) \mathrm{d} y\right| .
$$


TABLE 4: Four types of overlapping situations of cyclist domain in a bicycle lane.

$\begin{aligned} & \text { Type of } \\ & \text { event }\end{aligned}$
Cyclist domain overlapping diagrams of events
Active
passing
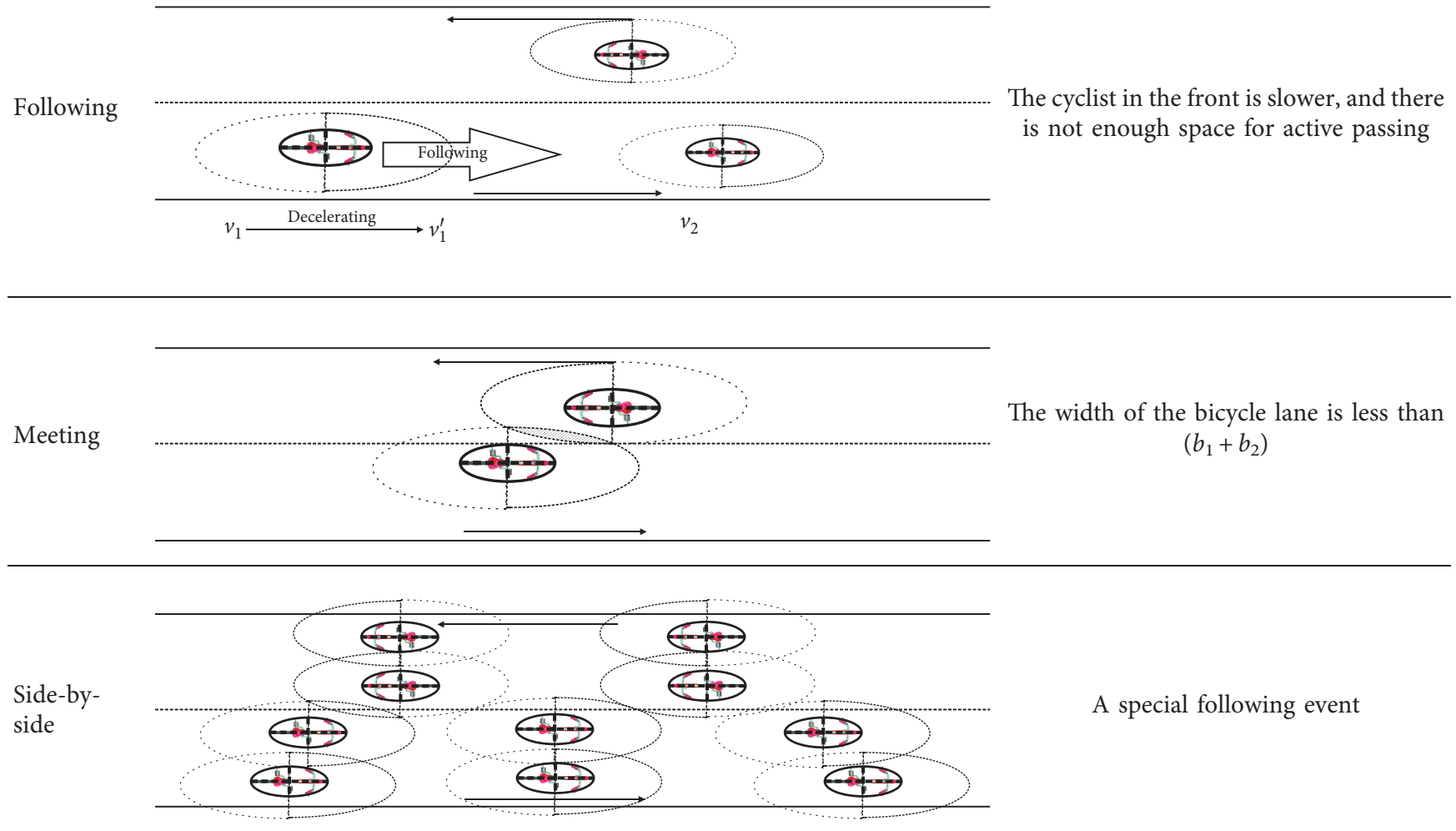

A special following event

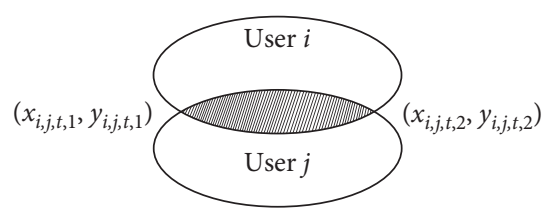

(a)

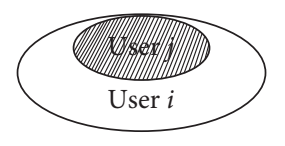

(c)

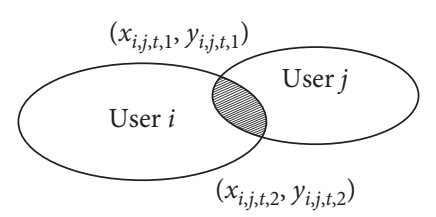

(b)

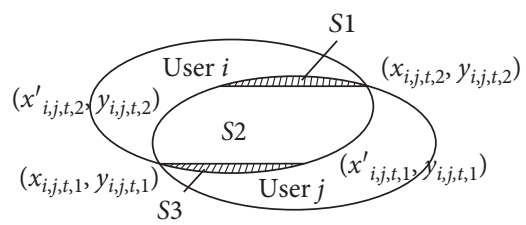

(d)

FIGURE 6: Four types of cyclist domain overlap. (a) Side overlap. (b) Longitudinal overlap. (c) Inclusion. (d) Unconventional overlap. 
The area of an inclusion region of two cyclist domains is then given by

$$
S_{t, i, j}=\pi \times a_{j, t} \times b_{j, t} .
$$

The area of an unconventionally overlapping region of two cyclist domains is written as

$$
\begin{aligned}
& S_{t, i, j}=S_{1}+S_{2}+S_{3}, \\
& \left\{\begin{aligned}
S 1 & =\left|\int_{x_{i, j, t, 2}^{\prime}}^{x_{i, j, t, 2}}\left(y_{j, t}+\frac{b_{j, t}}{a_{j, t}} \sqrt{a_{j, t}-\left(x-x_{i, t}\right)^{2}}-y_{2}\right) \mathrm{d} x\right|, \\
S 2 & =\mid \int_{y_{i, j, t, 1}}^{y_{i, j, t, 2}}\left(x_{i, t}+\frac{a_{i, t}}{b_{i, t}} \sqrt{b_{i, t}^{2}-\left(y-y_{i, t}\right)^{2}}\right) \\
& -\left(x_{j, t}-\frac{a_{j, t}}{b_{j, t}} \sqrt{b_{j, t}^{2}-\left(y-y_{j, t}\right)^{2}}\right) \mathrm{d} y \mid, \\
S 3 & =\left|\int_{x_{i, j, t, 1}}^{x_{i, j, t, 1}^{\prime}}\left(y_{1}-y_{i, t}+\frac{b_{i, t}}{a_{i, t}} \sqrt{a_{i, t}-\left(x-x_{i, t}\right)^{2}}\right) \mathrm{d} x\right|,
\end{aligned}\right.
\end{aligned}
$$

where $S_{t, i, j}$ is the area of the overlapping region of users $i$ and $j$ at the $t$ th time step; $x_{i, j, t, 1}, x_{i, j, t, 2}, y_{i, j, t, 1}$, and $y_{i, j, t, 2}$ are the coordinates of the two intersection points of the two cyclist domains at the th time step, which can be determined by the intersection points of the ellipse; $\left(x_{i, t}, y_{i, t}\right)$ and $\left(x_{j, t}, y_{j, t}\right)$ are the coordinates of cyclists $i$ and $j$ at the $t$ th time step; $a_{i, t}$ and $a_{j, t}$ are, respectively, the semimajor radii of the cyclist domains of users $i$ and $j$ at the $t$ th time step; $b_{i, t}$ and $b_{j, t}$ are, respectively, the semiminor radii of the cyclist domain of users $i$ and $j$ at the th the step; and the parameters $a_{i, t}, b_{i, t}, a_{j, t}$, and $b_{j, t}$ can be calculated by equation (1).

3.3. Users' Cyclist Domain Influence Ratio. We now focus on the CDIR. Based on the cyclist domain model and the calculation of cyclist-domain overlapping areas, we thus can define two important cyclist perception indices, the difference in the area of the cyclist domain of user $i$ before and after hindrance by user $j$ and the average area of the overlapping region of users $i$ and $j$. Notably, the areas of the cyclist domain overlap of different users cannot be summed without normalization because users' perceptions differ. The area of change and the overlap in cyclist domain are then normalized and denoted by a dimensionless index, the CDIR, $\omega_{i, j}$.

\subsubsection{The Difference in Area of the Cyclist Domain of User $i$} before and after Hindrance by User $j$. The average area of user $i$ 's cyclist domain is denoted by $\overline{S_{i}}$, which can be obtained by the area of an ellipse with semiaxes $a$ and $b$. The average area of the cyclist domain of user $i$ during invasion by user $j$ is denoted by $\overline{S_{i, j, a}}$, which can be calculated by equations (3)-(7).
Then, we can determine the difference of the cyclist domain area of user $i$ before and after hindrance by user $j$, $\Delta S_{i, j}$, by

$$
\Delta S_{i, j}=\left|\overline{S_{i, j, a}}-\overline{S_{i}}\right|
$$

3.3.2. The Average Area of Overlap between Users $i$ and $j$. Let $S_{t, i, j}$ denote the area of overlap between users $i$ and $j$ at the $t$ th time step during an overlapping process in $n$ time steps. Then, it is not difficult to calculate the average area of the overlapping domains of users $i$ and $j, \overline{S_{i, j, \text { inv }}}$, by

$$
\overline{S_{i, j, \text { inv }}}=\frac{\sum S_{t, i, j}}{n} .
$$

3.3.3. The CDIR of User $i$. Note that user $i$ may encounter hindrance from more than one users. We then calculate CDIR of user $i$ by

$$
\omega_{i, j}=\frac{\Delta S_{i, j}+\overline{S_{i, j, \text { inv }}}}{\overline{S_{i}}} .
$$

3.4. The Comfort of User $i$. The comfort of user $i$ is expressed as the summation of the products of the CDIR and its corresponding duration. Therefore, we have

$$
\operatorname{LOS}_{i}=\sum_{j} \omega_{i, j} \times t_{, i, j}
$$

where $\operatorname{LOS}_{i}$ is the comfort of user $i$ in the focal bicycle lane during the evaluation period; $\omega_{i, j}$ is the CDIR of user $i$ interacting with user $j$ during the period; and $t_{p, i, j}$ is the duration of time when two cyclists interact with each other, measured in seconds.

3.5. Bicycle Lane LOS Scores. For each cyclist, we can easily obtain his comfort score according to the calculation procedures given in Sections 3.2-3.4. Table 5 presents the calculation results for the data extracted from the aforementioned cycling video. The LOS of a bicycle lane is then defined as the average of all user comfort levels:

$$
\text { BicycleLOS }=\frac{\sum_{i}^{m} \operatorname{LOS}_{i}}{m},
$$

where $m$ is the number of users cycling in the bicycle lane during the evaluation period.

\section{Case Study}

4.1. Cycling Trajectory Extraction and Data Processing. We, in this section, present a case study to illustrate the workflows of our proposed methodology. The cycling data were collected from a bicycle lane under the Central Railway Station tunnel in 's-Hertogenbosch in the Netherlands. The bicycle lane is $60 \mathrm{~m}$ long and $2.75 \mathrm{~m}$ wide. 15,000 cyclists on 
TABle 5: Calculation sheet (a subset of users is shown).

\begin{tabular}{|c|c|c|c|c|c|c|c|c|c|c|c|c|}
\hline Users & Index & 1 & 2 & 3 & 4 & 5 & 6 & 7 & 8 & 9 & 10 & 11 \\
\hline \multirow[t]{2}{*}{1} & Dur. (s) & & 2.0 & & 3.5 & & & & & & & \\
\hline & CDIR & & 1.81 & & 1.11 & & & & & & & \\
\hline \multirow{2}{*}{2} & Dur. (s) & 2.0 & & 6.0 & & & & & & & & \\
\hline & CDIR & 1.02 & & 0.85 & & & & & & & & \\
\hline \multirow{2}{*}{3} & Dur. (s) & & 6.0 & & & & & & & & & \\
\hline & CDIR & & 1.11 & & & & & & & & & \\
\hline \multirow{2}{*}{4} & Dur. (s) & 3.5 & & & & & & & & & & \\
\hline & CDIR & 0.68 & & & & & & & & & & \\
\hline \multirow{2}{*}{5} & Dur. (s) & & & & & & 5.7 & & & & & \\
\hline & CDIR & & & & & & 0.61 & & & & & \\
\hline \multirow{2}{*}{6} & Dur. (s) & & & & & 5.7 & & & & & & \\
\hline & CDIR & & & & & 1.15 & & & & & & \\
\hline \multirow{2}{*}{7} & Dur. (s) & & & & & & & & 11.5 & & & \\
\hline & CDIR & & & & & & & & 0.90 & & & \\
\hline \multirow{2}{*}{8} & Dur. (s) & & & & & & & 11.5 & & & & \\
\hline & CDIR & & & & & & & 0.89 & & & & \\
\hline \multirow{2}{*}{9} & Dur. (s) & & & & & & & & & & & \\
\hline & CDIR & & & & & & & & & & & \\
\hline \multirow{2}{*}{10} & Dur. (s) & & & & & & & & & & & 11.0 \\
\hline & CDIR & & & & & & & & & & & 1.07 \\
\hline \multirow{2}{*}{11} & Dur. (s) & & & & & & & & & & 11.0 & \\
\hline & CDIR & & & & & & & & & & 1.23 & \\
\hline & Dur. (s) & & & & & & & & & & & \\
\hline$\cdots$ & CDIR & & & & & & & & & & & \\
\hline \multicolumn{2}{|c|}{ Comfort degree } & 4.43 & 10.26 & 5.08 & 3.87 & 8.60 & 4.57 & 10.22 & 10.38 & 0 & 13.54 & 11.78 \\
\hline \multicolumn{13}{|c|}{ BicycleLOS $=\left(\sum_{i}^{m} \operatorname{LOS}_{i} / n\right)=7.43$} \\
\hline
\end{tabular}

average pass the bicycle lane every day. The video was taken at the entrance of the Central Railway Station tunnel at 5:00 pm on Tuesday the 4th of September 2012.

Cycling trajectories for 22 users were extracted with image recognition technology, as shown in Figure 7, to be used for the LOS evaluation of bicycle lane. The cycling trajectory data include the cyclist's coordinates and instantaneous speeds every 0.5 second. The direction of the bicycle lane is parallel to the $x$-axis. The cyclist domains are calculated using equation (1).

4.2. Bicycle Lane LOS Evaluation Based on HCM 2010. We first evaluate the bicycle lane LOS by the HCM-2010 method:

$$
\begin{aligned}
\text { Bicycle LOS Score }= & 5.446-0.00809(E)-15.86(\mathrm{RW}) \\
& -0.287(\mathrm{CL})-0.5(\mathrm{DP}),
\end{aligned}
$$

where $E$ is the number of weighted events per minute, calculated as the sum of the number of meetings per minute and 10 times the number of events of active passing per minute; RW is the reciprocal of the path width in feet; CL equals 1 if the trail has a centre line and 0 otherwise; and DP is equal to either the number of events of delayed passing per minute or 1.5 , whichever is smaller.

Based on the HCM 2010 bicycle lane LOS equation (13), the following parameters and the bicycle lane LOS can be easily determined:

$$
\begin{aligned}
E & =0+10 \times 2, \\
\mathrm{RW} & =\frac{1}{2.75 \times 3.28}=0.111, \\
\mathrm{CL}= & 0, \\
\mathrm{DP}= & 0.5, \\
\text { BicycleLOS }= & 5.446-0.00809(E)-15.86(\mathrm{RW}) \\
& -0.287(\mathrm{CL})-0.5(\mathrm{DP})=3.274 .
\end{aligned}
$$

According to the classification criterion of HCM 2010 (Table 6), the LOS score falls into the " $\mathrm{C}$ " level.

\subsection{Bicycle Lane LOS Evaluation Based on Cyclist Domains}

4.3.1. Derive Each User's Cyclist Domain Data. Take User 1 as an example. First, we calculate the distance between User 1 and other users in the cycling direction and determine User 1's cyclist domain data from cycling trajectories (see Table 7 and Figure 8). Data for other cyclists during the evaluation period can be obtained by the same way.

4.3.2. Determine User i's Domain Overlaps and the Overlapping Duration during Which They Overlap. When the following conditions are met, there is an overlap between User 1 and another user. At a certain time step, the elliptic 


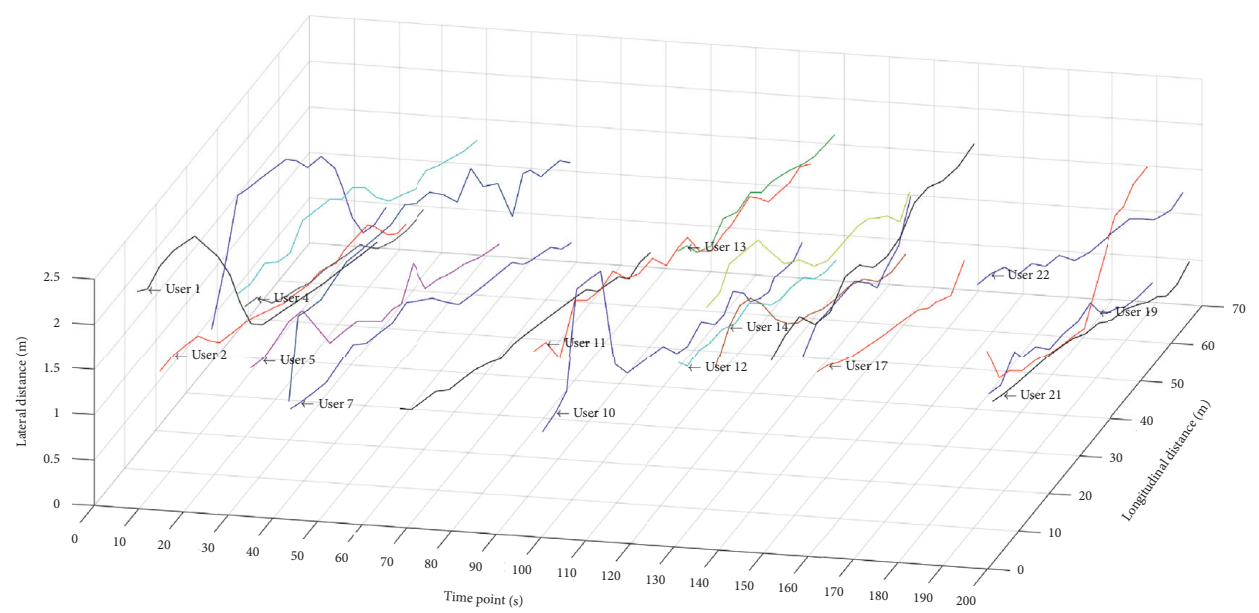

Figure 7: Cycling trajectories for 22 users.

TABLE 6: HCM-2010 criterion for bicycle lane LOS on shared-use and exclusive paths.

\begin{tabular}{lcc}
\hline LOS & LOS score & Comments \\
\hline A & $>4.0$ & Optimum conditions, ample ability to absorb more riders \\
$\mathrm{B}$ & $>3.5-4.0$ & Good conditions, some ability to absorb more riders \\
$\mathrm{C}$ & $>3.0-3.5$ & Meets current demand, marginal ability to absorb more riders \\
$\mathrm{D}$ & $>2.5-3.0$ & Many conflicts, some reduction in bicycle travel speed \\
$\mathrm{E}$ & $>2.0-2.5$ & Very crowded, with significantly reduced bicycle travel speed \\
$\mathrm{F}$ & $\leq 2.0$ & Significant user conflicts and diminished experience \\
\hline
\end{tabular}

TABLe 7: Cyclist domain data for User 1.

\begin{tabular}{|c|c|c|c|c|c|}
\hline Time step & $\begin{array}{l}\text { Cyclist domain of User } \\
1\left(\mathrm{~m}^{2}\right)\end{array}$ & $\begin{array}{c}\text { Area of overlapping region } \\
\left(\mathrm{m}^{2}\right)\end{array}$ & Time step & $\begin{array}{l}\text { Cyclist domain of User } \\
1\left(\mathrm{~m}^{2}\right)\end{array}$ & $\begin{array}{l}\text { Area of overlapping region } \\
\left(\mathrm{m}^{2}\right)\end{array}$ \\
\hline 19 & 13.919 & 3.332 & 15 & 5.63 & 1.798 \\
\hline 20 & 10.549 & 0.936 & 16 & 9.539 & 1.213 \\
\hline 21 & 12.788 & 4.073 & 18 & 8.607 & 3.112 \\
\hline \multirow[t]{4}{*}{22} & 15.46 & 5.967 & 19 & 13.919 & 5.756 \\
\hline & & & 20 & 10.549 & 5.446 \\
\hline & & & 21 & 12.788 & 0.289 \\
\hline & & & 22 & 15.46 & 3.674 \\
\hline Duration (s) & $\begin{array}{l}\text { Average area of cyclist } \\
\text { domain }\left(\mathrm{m}^{2}\right)\end{array}$ & $\begin{array}{c}\text { Average area of overlapping } \\
\text { region }\left(\mathrm{m}^{2}\right)\end{array}$ & Duration (s) & $\begin{array}{c}\text { Average area of cyclist } \\
\text { domain }\left(\mathrm{m}^{2}\right)\end{array}$ & $\begin{array}{c}\text { Average area of overlapping } \\
\text { region }\left(\mathrm{m}^{2}\right)\end{array}$ \\
\hline 2 & 13.179 & 3.577 & 3.5 & 10.927 & 3.041 \\
\hline
\end{tabular}

equations of the two users' cyclist domains has a real root, or the elliptic equations of the two users' cyclist domains has no real root, but the ellipse's centre of User 1 is located in the ellipse of another user. Through calculation and analysis, overlapping regions can be seen between User 1 and User 2, as well as User 1 and User 4.

4.3.3. Calculate the Average Area of Overlap in the Cyclist Domain of User $i$ with Other Users. We firstly use equation (1) to calculate the change in the cyclist domain area before and after each event. Then, we determine the overlapping area of user $i$ with other users by equations (3)-(7) and then calculate the average overlapping area in the cyclist domain of user $i$ with other interacting users.

For example, User 1 overlapped with User 2 from the 19th time step to the 22nd time step, with an average overlap of

$\overline{S_{p, 1,2, \text { inv }}}=\frac{3.332+0.936+4.073+5.967}{4}=3.577 \mathrm{~m}^{2}$.

Next, consider Users 1 and 4, who overlapped from the 15 th to the 22 nd time step. The average overlapping area of Users 1 and 4 is

$$
\overline{S_{f, 1,4, \text { inv }}}=3.041 \mathrm{~m}^{2} \text {. }
$$




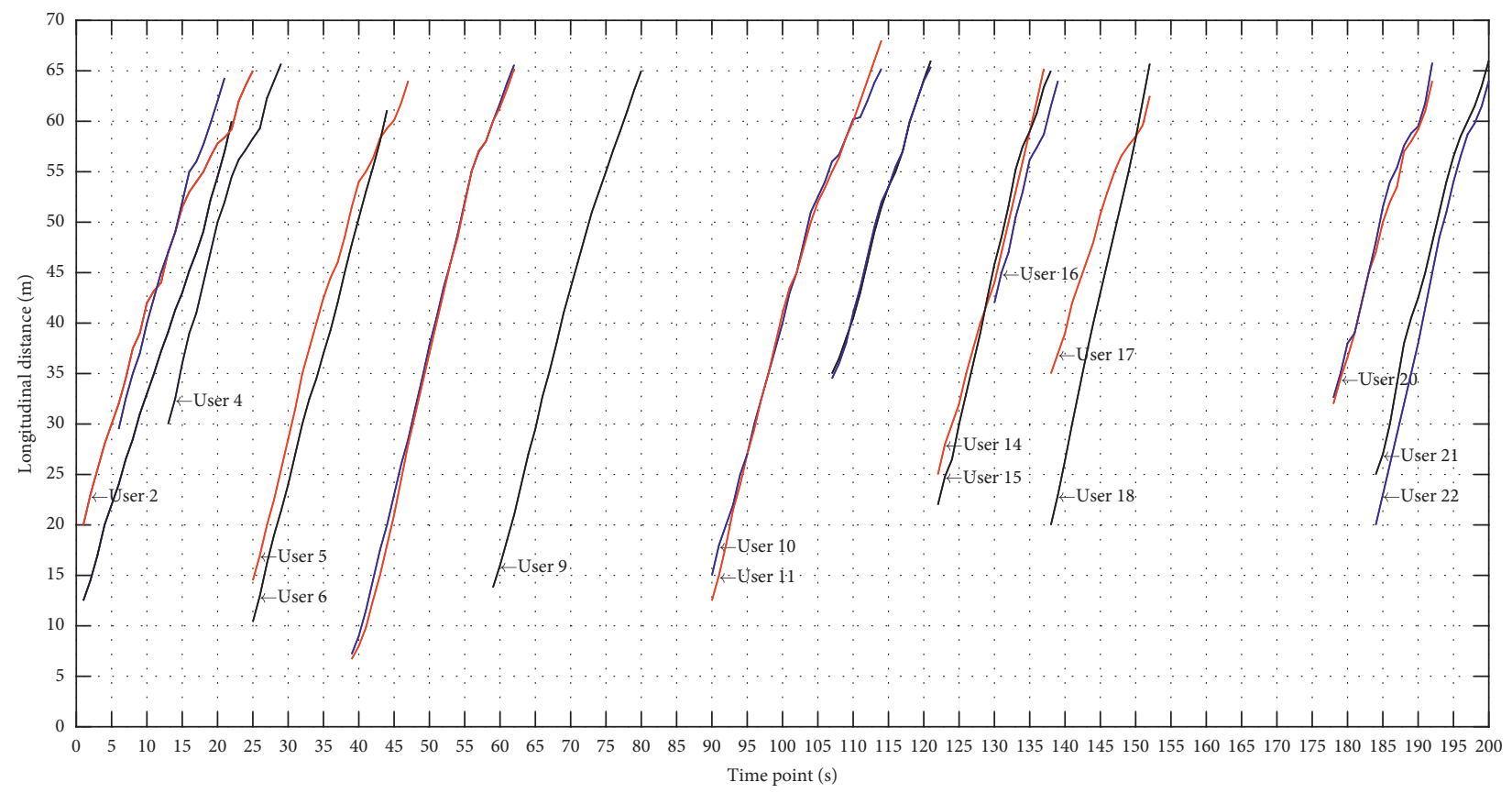

FIgURE 8: Longitudinal distance graph.

TABle 8: Proposed LOS criteria for bicycle lanes based on cyclist domain.

\begin{tabular}{lcc}
\hline LOS & LOS score & Comments \\
\hline A & $<6.5$ & Optimal conditions, ample ability to absorb more riders \\
$\mathrm{B}$ & $6.5-7$ & Good conditions, some ability to absorb more riders \\
$\mathrm{C}$ & $>7-7.5$ & Meets current demand, marginal ability to absorb more riders \\
$\mathrm{D}$ & $>7.5-8$ & Many conflicts, some reduction in bicycle travel speed \\
$\mathrm{E}$ & $>8-8.5$ & Very crowded, with significantly reduced bicycle travel speed \\
$\mathrm{F}$ & $>8.5$ & Significant user conflicts and diminished experience \\
\hline
\end{tabular}

4.3.4. Calculate the CDIR of User $i$. The average speed of User 1 was $4.13 \mathrm{~m} / \mathrm{s}$, and the average area of User 1's cyclist domain was $8.3 \mathrm{~m}^{2}$. During the period of overlap with User 2 , the difference in the area of User 1's cyclist domain is given by

$$
\Delta S_{1,2}=|13.179-8.3|=4.879 m^{2} .
$$

Similarly, during the period of overlap with User 4, the difference in the area of User 1's cyclist domain is

$$
\Delta S_{1,4}=|10.927-8.3|=2.627 \mathrm{~m}^{2} .
$$

According to equation (12), the CDIR of User 1 interacted with User 2 is

$$
\omega_{1,2}=\frac{4.879+3.577}{8.3}=1.019 \text {. }
$$

The CDIR of User 1 interacted with User 4 is

$$
\omega_{1,4}=\frac{2.627+3.041}{8.3}=0.683 \text {. }
$$

By the same procedures, we can calculate the CDIR of a particular user with all other encountered users in the bicycle lane.
4.3.5. Calculate the Comfort Level of User i. Take User 1, for example. According to equation (12), the comfort level of User 1 is

$$
\operatorname{LOS}_{1}=\omega_{1,2} \times t_{1,2}+\omega_{1,4} \times t_{1,4}=1.019 \times 2+0.683 \times 3.5=4.428 .
$$

4.3.6. Determine the Bicycle Lane LOS Score. Table 7 gives the calculation results in terms of the comfort level for each cyclist and the LOS of the bicycle lane:

$$
\text { BicycleLOS }=7.4298
$$

4.4. Proposed LOS Criteria for Bicycles on Shared-Use and Exclusive Paths. According to the LOS criteria for bicycles on shared-use and exclusive paths (Table 6), the LOS of the bicycle lane is $\mathrm{C}$, with a score of 7.43. In view of the LOS criteria used in HCM 2010, we specify cyclist-domain-based LOS criteria for bicycle lanes, shown in Table 8. 


\section{Concluding Remarks}

In this paper, we present a cyclist-domain-based method for evaluating the LOS for bicycle lanes. Describing the bicycle lane LOS from a user perception's perspective has been accepted in the previous studies, but without applying the cyclist-domain concept, it would be a real challenge and probably the first time to report on such a method to successfully describe bicycle lane LOS. With the use of data collected from video recording at selected bicycle lanes, we set up a cyclist-domain model. We also identified the cycling interactions and classified their impacts into four kinds of cyclist domain situations. For a particular cyclist, the external impact from other encountered users is characterized by the CDIR, which extensively describe cyclists' perception. The proposed method performed better because it explicitly considered the cycling characteristic.

Although the proposed method is motivated by current practice in the cycling transport system, a nonnegligible limitation deserves further investigation. Our paper makes an analysis of cyclist domain based on limited datasets. We need to justify the method, especially the LOS criteria, by enriching the cycling sample and testing more scenarios under various traffic conditions, e.g., raining and uneven pavement.

\section{Data Availability}

All data, models, and code generated or used during the study appear in the submitted article.

\section{Conflicts of Interest}

The authors declare that there are no conflicts of interest regarding the publication of this paper.

\section{Acknowledgments}

This research was substantially supported by the research grants from the National Natural Science Foundation Council of China (51978082), the grants from the International cooperation and expansion program of "Double First-Class" scientific research of Changsha University of Science and Technology (2019IC10), and the grant of Degree and Postgraduate Education Reform Project of Hunan Province (2019JGYB169).

\section{Supplementary Materials}

The velocity and space data were obtained from a released cycling traffic video filmed at the intersection of Vredenburg, Lange Viestraat, and Sint Jacobsstraat, on the edge of Utrecht Central Station. The video was taken during morning peak hour in April 2014. Data of 20 cyclists were extracted from the video in 0.5-second intervals, including real-time speed and adjacent cyclists' polar coordinates. (Supplementary Materials)

\section{References}

[1] D. Sims, S. A. Matthews, M. J. Bopp, L. S. Rovniak, and E. Poole, "Predicting discordance between perceived and estimated walk and bike times among university faculty, staff, and students," Transportmetrica A: Transport Science, vol. 13, no. 2, pp. 91-107, 2018.

[2] A. Ermagun and D. Levinson, "Public transit, active travel, and the journey to school: a cross-nested logit analysis," Transportmetrica A: Transport Science, vol. 13, no. 1, pp. 2437, 2017.

[3] P. Angeloudis, J. Hu, and M. G. H. Bell, "A strategic repositioning algorithm for bicycle-sharing schemes," Transportmetrica A: Transport Science, vol. 10, no. 8, pp. 759-774, 2014.

[4] I. A. Forma, T. Raviv, and M. Tzur, "A 3-step math heuristic for the static repositioning problem in bike-sharing systems," Transportation Research Part B: Methodological, vol. 71, pp. 230-247, 2015.

[5] Y. Li, W. Y. Szeto, J. Long, and C. S. Shui, "A multiple type bike repositioning problem," Transportation Research Part B: Methodological, vol. 90, pp. 263-278, 2016.

[6] S. C. Ho and W. Y. S. Szeto, "A hybrid large neighborhood search for the static multi-vehicle bike-repositioning problem," Transportation Research Part B: Methodological, vol. 95, pp. 340-363, 2017.

[7] X. Jia, M. O’Mara, and M. Guan, "Rethinking geometric design standards for bike paths," Journal of Transportation Engineering, vol. 133, no. 9, pp. 539-547, 2007.

[8] J. Duthie and A. Unnikrishnan, "Optimization framework for bicycle network design," Journal of Transportation Engineering, vol. 140, no. 7, Article ID 4014028, 2014.

[9] C.Y. Duan, D. Hu, and X. Chen, "Optimizing the layout of bike-sharing rental stations based on the hierarchical model," in Proceedings of the 16th COTA International Conference of Transportation Professionals, American Society of Civil Engineers, Shanghai, China, July 2016.

[10] Q. Chen and T. Sun, "A model for the layout of bike stations in public bike-sharing systems," Journal of Advanced Transportation, vol. 49, no. 8, pp. 884-900, 2015.

[11] A. Faghih-Imani and N. Eluru, "Examining the impact of sample size in the analysis of bicycle-sharing systems," Transportmetrica A: Transport Science, vol. 13, no. 2, pp. 139-161, 2017.

[12] B. W. Landis, "Bicycle system performance measures," ITE Journal,vol. 66, no. 2, pp. 18-26, 1996.

[13] K. Kang and K. Lee, "Development of a bicycle level of service model from the user's perspective," KSCE Journal of Civil Engineering, vol. 16, no. 6, pp. 1032-1039, 2012.

[14] X. Liang, M. Xie, and X. Jia, "Use of entropy to analyze level of service of dedicated bike lanes in China," Advancesin Mechanical Engineering, vol. 9, no. 6, pp. 1-12, 2017.

[15] H. Botma, "Method to determine levels of service for bicycle paths and pedestrian-bicycle paths," Transportation Research Record, vol. 1502, pp. 38-44, 1995.

[16] J. E. Hummer, N. M. Rouphail, J. L. Toole et al., "Evaluation of safety, design, and operation of shared-use paths: final report," Report FHWA-HRT-05-137, Federal Highway Administration, Washington, DC, USA, 2006.

[17] Transportation Research Board, Highway Capacity Manual, Transportation Research Board, Washington, DC, USA, 2010.

[18] R. Sommer, Personal Space: The Behavioral Basis of Design, Prentice-Hall, Englewood Cliffs, NJ, USA, 1969. 
[19] S. Kim, J. Choi, S. Kim, and R. Tay, "Personal space, evasive movement and pedestrian level of service," Journal of Advanced Transportation, vol. 48, no. 6, pp. 673-684, 2014.

[20] E. M. Goodwin, “A statistical study of ship domains," Journal of Navigation, vol. 28, no. 3, pp. 328-344, 1975.

[21] M. J. Horowitz, D. F. Duff, and L. O. Stratton, "Body-buffer zone: exploration of personal space," Archivesof General Psychiatry, vol. 11, pp. 651-656, 1964. 